\title{
A Cadaveric Study of Occurrence of Extralaryngeal Branches of the Recurrent Laryngeal Nerve with Reference to its Importance in Thyroid Surgery
}

\section{Shantanu Nandy, ${ }^{1}$ Anirban Banerjee, ${ }^{2}$ Saumik Das ${ }^{3}$}

\begin{abstract}
Introduction:
Recurrent Laryngeal Nerve Palsy (RLNP) is the most common complication of thyroid surgery. Patients complain of hoarseness of voice and sometimes variable amount of respiratory distress due to palsy of recurrent laryngeal nerve (RLN), extra laryngeal branches of which are often ligated along with Inferior Thyroid Artery (ITA) during thyroidectomy. So prevalence and variable pattern of extra laryngeal nerve branches (ELNB) of RLN should be known to the thyroid surgeon to avoid post operative complications.

Materials and Methods

Dissection was carried on the neck of properly embalmed 25 adult cadavers including both sexes in the dissection hall to study extra laryngeal nerve branches (ELNB) of 50 RLN specimens.

$\underline{\text { Results }}$

Out of 50 RLN, 13 nerves (26\%) have ELNBs. Only in 4 cadavers (16\%) they are bilateral. In 9 cases RLN branches were surrounding either single trunk of ITA or branches of the artery. In rest of the 4 cases ELNBS were passing entirely either in front or behind the ITA.

Conclusion

The gold standard for preservation of the RLN during thyroid surgery is still visual anatomical identification. Proper dissection and anatomical identification of the RLN and all its branches is very important prior to the clamping of the ITA and all its branches.

$\underline{\text { Kevwords: }}$

Recurrent Laryngeal Nerve; Thyroidectomy; Cadaver
\end{abstract}

ABSTRACT

$\mathrm{T}$ There are many thyroid diseases where only pharmacological treatment is not sufficient and they require thyroidectomy. During the surgery, surgeon has to ligate both superior thyroid artery and inferior thyroid artery (ITA). As the ITA has variable

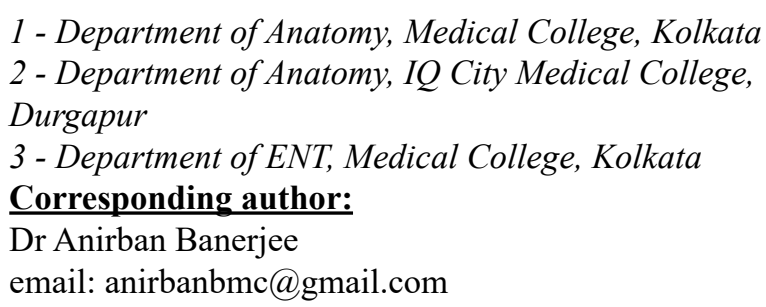

relationships with Recurrent Laryngeal nerve (RLN) in trachea - oesophageal groove close to the lower pole of thyroid gland, the nerve becomes vulnerable while ligating the artery. Thus in post thyroidectomy patients recurrent laryngeal nerve palsy (RLNP) is a common complication. ${ }^{1}$ So a surgeon must be aware of the variable relationship of RLN and ITA close to the lower pole of thyroid gland to avoid RLNP. ${ }^{2}$

The situation becomes more complicated for the surgeon if the RLN has extra laryngeal branches. In most of the cases the RLN trunk enters into the larynx after crossing the ITA and ramifies within the larynx. But it is 


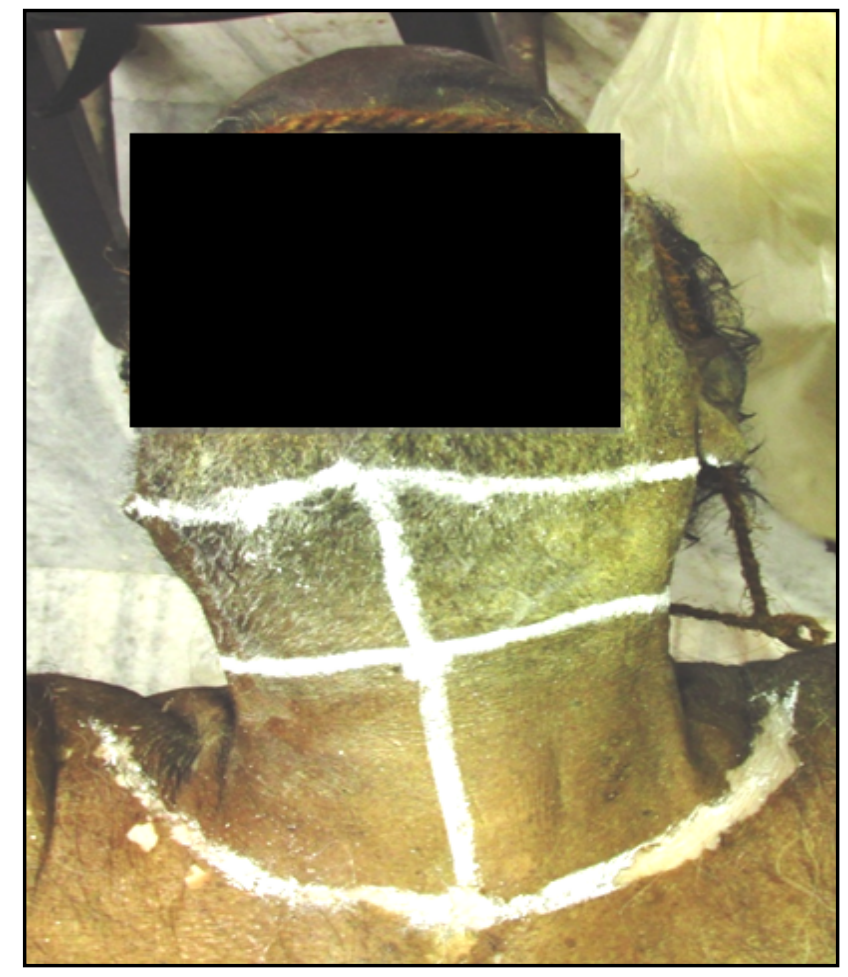

Fig. 1. Incision lines drawn on the neck of cadaver

noticed that, while passing behind the cricothyroid joint RLN is often divided into 2 or 3 branches outside the larynx in relation with ITA. Injury to a small twig of these extra laryngeal nerve branches (ELNB) may result in voice changes. ${ }^{6}$ In these cases surgeon requires very meticulous approach and precision to restore the nerve branches by identifying and exposing the nerve itself and all of its branches. ${ }^{3,4}$

\section{Method and material}

A cross sectional study was conducted where right and left side of the neck of 25 properly embalmed donated cadavers were dissected within a span of 2 years. Both male and female adult cadavers were dissected. Only fresh cadavers were chosen excluding the : a) cadavers which were already dissected, b) cadavers with scar in the neck, c) cadavers with prior neck surgery.

Cadavers were kept in supine position on dissection table and cervical region were extended. Incision lines were drawn by chalk. A transverse incision was made at

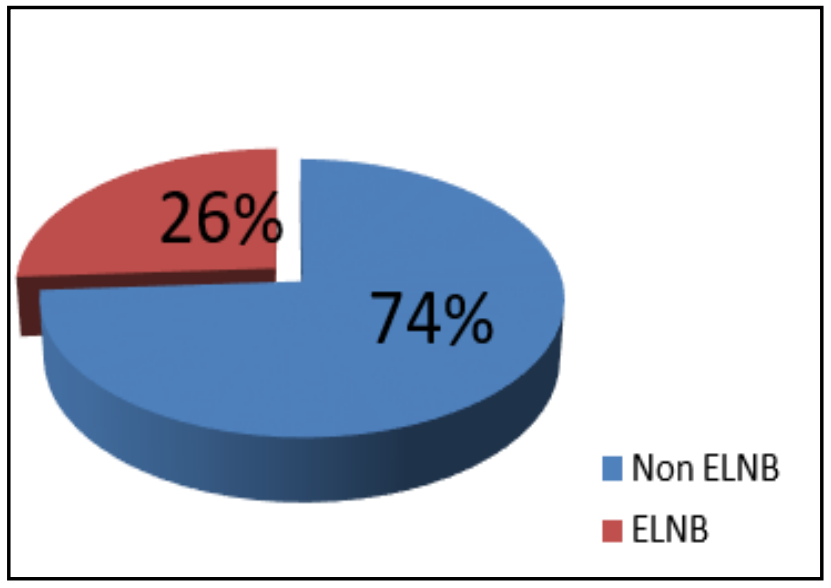

Fig. 2. 26\% RLNs having extra laryngeal branches among 50 nerve specimens

the level of upper border of thyroid cartilage joining the posterior margins of the sternocleidomastoid muscles of both sides. Another transverse incision was made along upper border of clavicle passing through the jugular notch. Midpoints of these 2 incisions are joined by a vertical incision along midline. Sometimes if necessary, over exposure was done by dissecting the sub mandibular region. (Fig.1) After retracting skin, superficial fascia along with platysma, superficial structures including anterior jugular veins and the anterior strap muscles of the neck were dissected and retracted.

Sternocleidomastoid (SCM) muscle, Carotid arteries and internal jugular vein were exposed and were retracted to explore the vagus nerve in between and deep to the artery and the vein to point out the origin of RLN. Then the lower pole of thyroid gland was retracted along with the trachea just deep to the gland. In the tracheo oesophageal (TE) groove in between lateral surface of the trachea and oesophagus, we searched for the RLN lying in close relation with ITA. The nerve is traced upto its point of origin for confirmation. ${ }^{5}$ Thyroid gland was kept in situ to avoid injury of the vessels and nerves.

\section{Results}

Among the 25 cadavers dissected most of were male constituting $68 \%$ (17) and rest of $32 \%$ (8) were female. We got 50 nerve specimens, of which 13 RLNs were divided into branches before entering the larynx. So the 


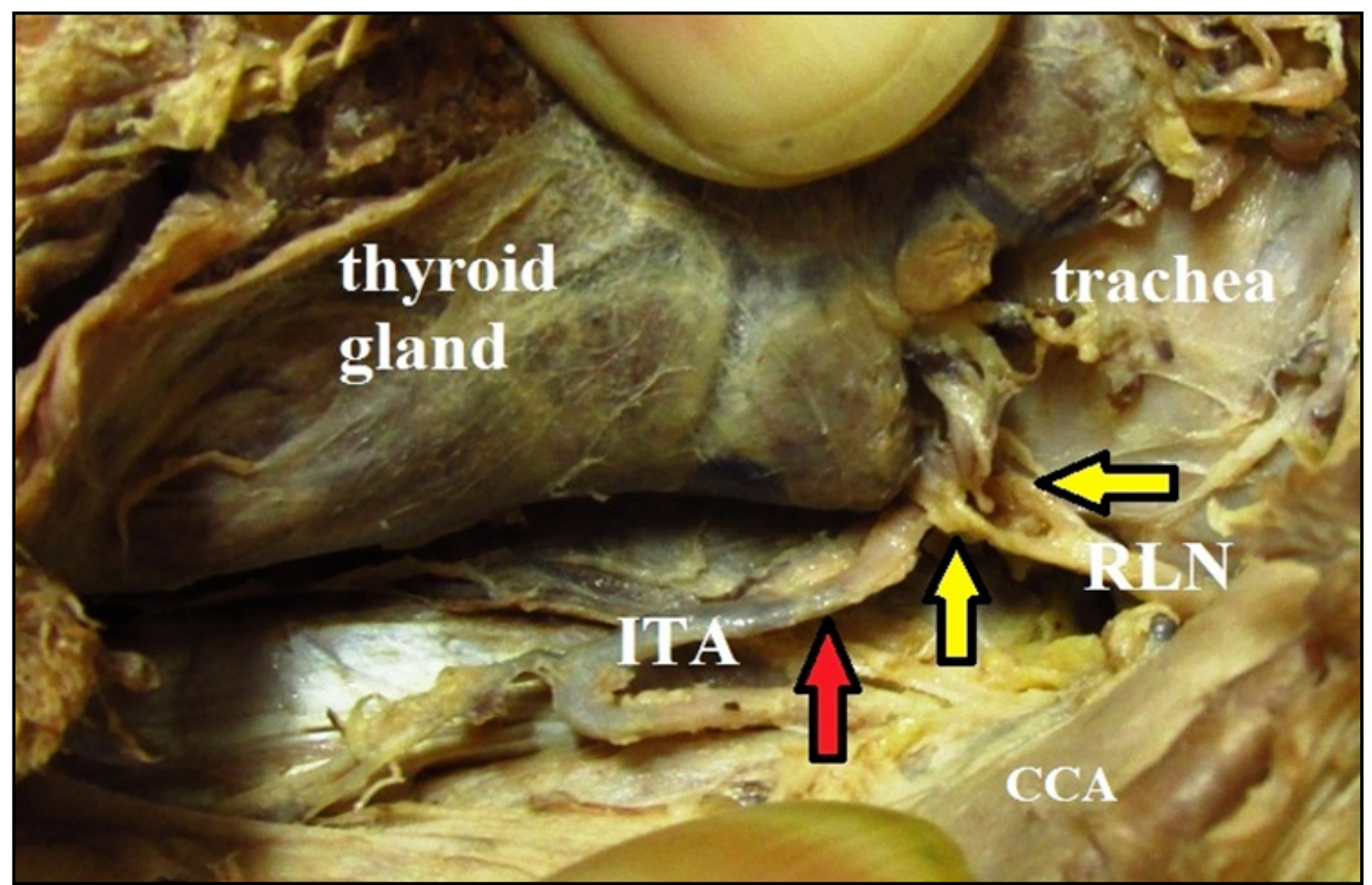

Fig. 3. Extralaryngeal nerve branches of RLN (yellow arrow) surrounding single trunk of ITA (red arrow). CCA- common carotid artery.

occurrence of extra-laryngeal nerve branches (ELNB) in our study is $26 \%$ (13 of 50). (Fig. 2) In these specimens the RLN is bifurcated or trifurcated. But not all of them were intermingling with the branches of ITA. In nine (9) cases RLN branches were surrounding either single trunk of ITA or branches of the artery. (Fig. 3) In rest of four (4) cases, RLNs with its branches are passing either anterior to or posterior to the ITA as a whole without intermingling with each other. (Fig. 4)

The 13 nerves with ELNB were found in 9 cadavers. So $36 \%$ (9 of 25 ) cadavers presented with ELNB. Among them bilateral ELNB are present in 4 cadavers $(16 \%)$ only.

\section{Discussion}

The variable relationship between RLN and ITA is a topic of research since the time of Theodor Kocher, who is known as the father of thyroid surgery. ${ }^{6}$ The ultimate goal of every researcher and thyroid surgeon is to reduce the rate of post thyroidectomy RLNP and thus to restore the voice and airway of the patients. According to the 'Farquharson's textbook of operative general surgery', in cases of bilateral RLNP during thyroidectomy, permanent tracheostomy is the way to restore airways unless the damage is no more than a temporary neuropraxia. ${ }^{7}$ It reflects why we are so concern about RLN and its damage.

This potentially catastrophic complication of thyroid surgery can occur permanently in $0.3-3 \%$ of cases, with transient palsies in 5-8\%. ${ }^{8}$ Dr Frank Lahey was first to introduce some improvised method for ligation of ITA, by which he recorded a nerve palsy rate of only $0.3 \%{ }^{6,9}$

But still the overall rate of RLNP is high. According to Titche, thyroidectomy accounts for $35.71 \%$ of surgical causes of injury to the RLN, and accounts for $3.73 \%$ of all causes. ${ }^{10}$ In the various studies the incidence of injury to the RLN in thyroidectomies ranges from 0.5 to $12 \%$. Frequency of this injury is more while ligating ITA and its branches. ${ }^{11,12,13}$

To change this grievous scenario constant emphasis is given on variation of RLN and its extra laryngeal 


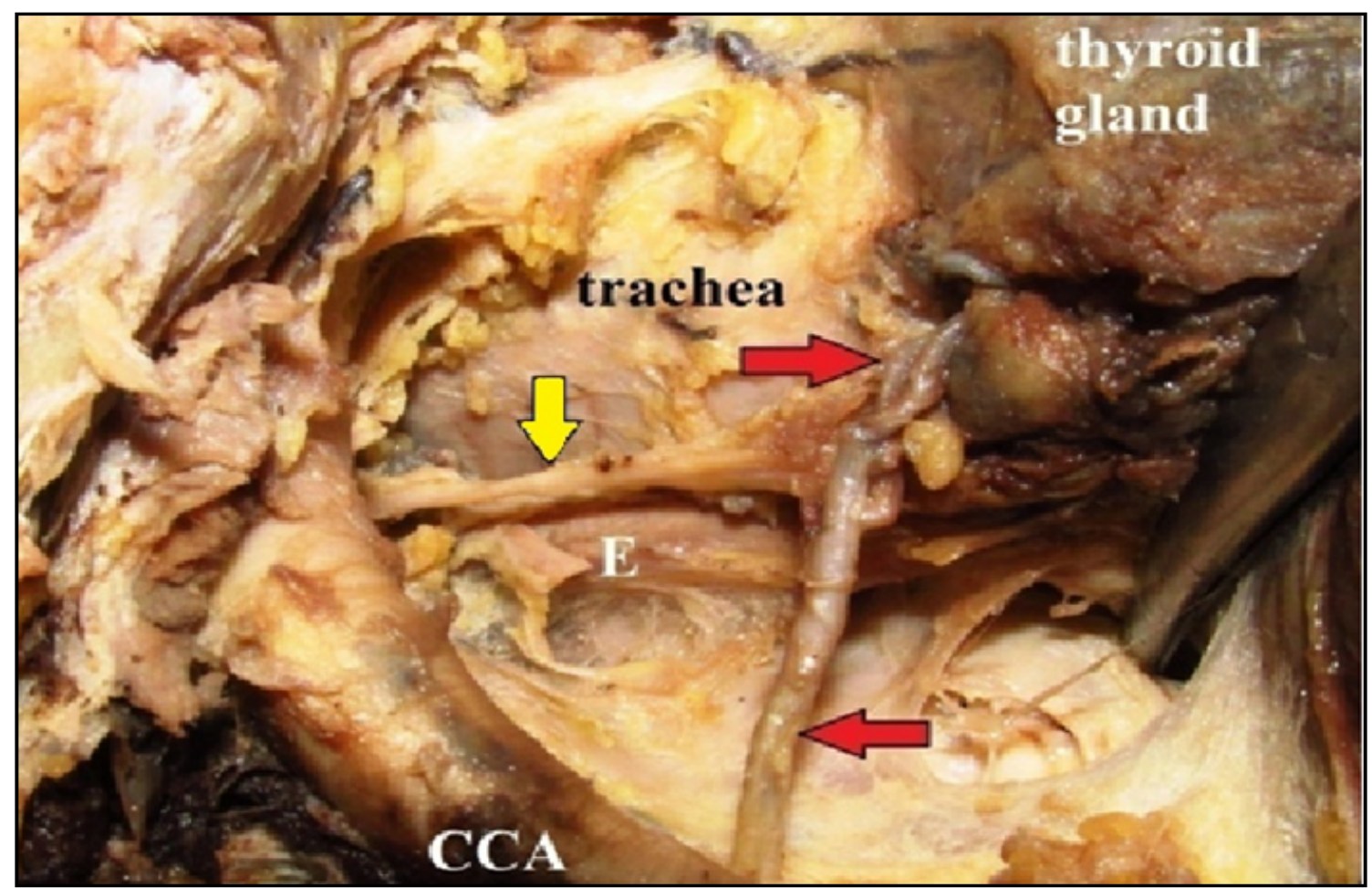

Fig. 4. FAN SHAPED extralaryngeal nerve branches of RLN (yellow arrow) GOING ENTIRELY BEHIND the ITA branches (red arrow). CCA- common carotid artery.

branches. A study by Ozer Makay et al. on 253 adult patients undergoing thyroid surgery revealed $24.1 \%$ RLNs are either bifurcated or trifurcated, which is close to our data $26 \% .{ }^{14}$ Among 57 RLNs which were bifurcating, only $17.5 \%(\mathrm{n}=10)$ were bilateral, which is also very close to the data we got $(16 \%$ cadavers with bilateral ELNB). Though there are other studies in which occurrence of RLN is either more or less.

In 3 separate studies by Nemiroff and Katz et al. 153,1177 , and 721 RLNs were observed of which $41.2 \%, 63 \%$ and $58 \%$ cases ELNBs were reported respectively. ${ }^{15,16,17}$ A study by Kandil et al. in 99 patients undergoing thyroidectomy 137 RLNs were observed among which 46 RLNs (34\%) bifurcated as ELNBs among which bilateral bifurcation occurred in 12 (27\%) nerves. ${ }^{18}$

On the other hand, Jacob et al. found that ELNB (bifurcation) was present in $14.8 \%$ cases. The study also noticed that $44.4 \%$ of the ITA was branched in two. This crucial and difficult situation has a higher likelihood of nerve injury if the surgeon is not aware of the branching variations of the ITA and the RLN. ${ }^{19}$

In an Indian study by Pradeep et al. on 584 RLNs, two ELNBs in $29.11 \%$ cases and three ELNBs in $1.36 \%$ cases were reported, which is close to our study result. ${ }^{20}$

This similarity points towards a regional specificity of ELNB which should be a research topic for future researcher. But due to less number of data we could not confirm it.

The variable rate of prevalence of ELNB of RLN reflects that it is very difficult to draw any particular pattern of occurrence of ELNBs, ultimately leaving no way for the surgeons to be more cautious about the RLN and its branches during ITA ligation.

But it is evident that regarding extralaryngeal branching of RLN, bifurcation is the most common type so far reported. According to 'Gray's Anatomy' and 'Last's Textbook of Anatomy' - whenever RLN is bifurcating outside the larynx, the anterior branch is mainly motor while the posterior branch is mainly sensory. ${ }^{21,22}$ Researchers also established the fact by IONM (intra operative nerve monitoring) method that 
in almost $100 \%$ cases anterior branch of bifurcated RLN has the motor fibres and the posterior branch carries sensory fibres. ${ }^{18,23}$

Contrary to this belief, some researchers using modern nerve signal recording device, recorded $1.3 \%$ to $11.5 \%$ of posterior ELNBs carry motor fibres along with the anterior branch. ${ }^{24,25}$ This striking difference of results between old and new studies is achieved definitely by using more sensitive machines to record motor nerve signal. It is a very significant finding as it will compel the surgeons to take care of both of the ELNBs and not to give the emphasis only on the anterior branch, which will ultimately reduce the prevalence of RLNP.

\section{Conclusion}

So even after trying various methods like IONM (intra operative nerve monitoring), the gold standard for preservation of the RLN during thyroid surgery is still visual anatomical identification. Proper dissection and anatomical identification of the RLN and all its branches is very important prior to the clamping of the ITA and all its branches. Other techniques which aim to preserve the RLN may be used only as an adjunct to the gold standard. In a setting where advanced technologies are not available, the surgeon must rely on the knowledge about the variations in the neurovascular anatomy of the thyroid gland to prevent complications of surgery. ${ }^{19,26,27}$

\section{References}

1. Ludlow CL, Bielamowicz SA. Neurogenic disorders of the larynx. Chapter 79. In : Snow B James Jr, Wackym P Ashley,Eds. Ballenger's Otorhinolaryngology. 17th edition. Shelton, Connecticut. B C Decker Inc / People's Medical Publishing House. 2009. p 916

2. Suzuki M, Kirchner JA, Murakami Y. The cricothyroid as a respiratory muscle: Its characteristics in bilateral Recurrent Laryngeal Nerve Paralysis. Ann Otol Rhinol Laryngol. 1970; 79: 976-83

3. Giovanni SD, Clawdia A, Agala $\mathrm{T}$ et al. The recurrent laryngeal nerve related to thyroid surgery. The American J Surgery 1999; $177: 485-8$

4. Cummings WC, Fredrickson MJ, editors. Otorhinolaryngology : Head and Neck Surgery. 3rd ed. Vol 3. USA. Mosby. 1998. P. 2445 9

5. G J Romanes,editor. Cunningham's Manual of Practical Anatomy. 15th ed (Reprint). Vol 3. Oxford. Oxford University Press.
2006. p. $65-9$

6. Giddings AE. The history of Thyroidectomy. J R Soc Med. 1998; 91 (Supp33) 3-6

7. Farquharson M, Moran B. Farquharson's textbook of operative general surgery. 9th edition. Great Britain (London). Hodder Education. 2005. p163-8

8. Hayward NJ, Grodski S, Yeung M, Johnson WR, Serpell J. Recurrent laryngeal nerve injury in thyroid surgery: a review. ANZ Journal of Surgery [cited 18th September,2012]. DOI: 10.1111/j.1445-2197.2012.06247.x

9. Harold Ellis: A History of Surgery. 2001, Chapter 13. p 199205

10. Titche LL. Causes of recurrent laryngeal nerve paralysis. Arch Otolaryngol. 1976; 102: 259-61

11. Karlan MS, Catz B, Dunkelman D et al. A safe technique for thyroidectomy with complete nerve dissection and parathyroid preservation. Head Neck Surgery 1984; 6: 1014-9

12. Max MH, Scherm M, Bland KI. Early and late complications after thyroid operations. South Med J. 1983; 76(8): 977-80

13. Riddell VH. Injury to recurrent laryngeal nerves during thyroidectomy - a comparison between the results of identification and non-identification in 1022 nerves exposed to risk. Lancet 1956; $2: 638-41$

14. Ozer M, Gokhan I, Mustafa Y, Mahir A, Enis Y. The recurrent laryngeal nerve and the inferior thyroid artery-anatomical variations during surgery. Langenbecks Arch Surg. 2008. 393:681-5 15. Nemiroff PM, Katz AD. Extralaryngeal divisions of the recurrent laryngeal nerve. Surgical and clinical significance. Am J Surg. 1982. 144:466-9

16. Katz AD, Nemiroff P. Anastamoses and bifurcations of the recurrent laryngeal nerve-Report of 1177 nerves visualized. Am Surg. 1993. 59:188-91

17. Katz AD. Extralaryngeal division of the recurrent laryngeal nerve. Report on 400 patients and the 721 nerves measured. Am J Surg. 1986. 152:407-10

18. Kandil E, Khalek MA, Aslam R, Friedlander P, Slakey D, Bellows CF. Recurrent laryngeal nerve: Significance of the anterior extralaryngeal branch. Surgery 2011; 149(6): 820-4

19. Jacob SM, Karen JPD, Ryner JCC. The Recurrent Laryngeal Nerve in Relation to the Inferior Thyroid Artery in Adult Filipino Cadavers. Philippine Journal Of Otolaryngology-Head And Neck Surgery $2011 ; 26(2): 13-7$

20. Pradeep PV, Jayashree B, Harshita SS. A closer look at laryngeal nerves during thyroid surgery: a descriptive study of 584 nerves. Anat Res Int. 2012;2012:490390

21. Stranding S, editor in chief. Gray's Anatomy. 40th edition. Churchill Livingstone Elsevier. 2008. Chapter 34. Page 577-93

22. Sinnatamby CS, editor. Last's Anatomy : Regional and applied. 12th edition. Churchill Livingstone Elsevier. 2011

23. Serpell JW, Yeung MJ, Grodski S. The motor fibers of the recurrent laryngeal nerve are located in the anterior extralaryngeal 
branch. Ann Surg. 2009. 249:648-52

24. Barczyński M, Stopa M, Konturek A, Nowak W. The overwhelming majority but not all motor fibers of the bifid recurrent laryngeal nerve are located in the anterior extralaryngeal branch. World J Surg. 2016; 40(3):629-35. doi: 10.1007/s00268-015-3257-4 25. Gurleyik E. Location of motor fibers within branches of the recurrent laryngeal nerve with extralaryngeal terminal bifurcation; Functional identification by intraoperative neuromonitoring. Surgery

\section{5; 158(5):1339-44}

26. Campos BA, Henriques PRF. Relationship between the recurrent laryngeal nerve and the inferior thyroid artery: a study in corpses. Rev. Hosp. Clín. Fac. Med. Sao Paulo. 2000; 55(6):195-200 27. Thomusch O, Sekulla C, Timmermann W, Neumann HJ, Kruse E, Muhlig HP, et al. Intraoperative neuromonitoring in thyroid surgery - Results of the German prospective multicentre study. Eur. Surg. 2003; 35(5):240-5. 